\title{
LITTÉRATURE
}

\author{
LUC FRAISSE
}

Université Marc Bloch de Strasbourg

\section{LE DISCOURS AUX MORTS DANS LA GUERRE DE TROIE N'AURA PAS LIEU DE GIRAUDOUX}

Abstract. Fraisse Luc, Le discours aux morts dans "La guerre de Troie n'aura pas lieu " de Giraudoux [The Speech to the Dead in Jean Giraudoux's « The Trojan War Will Not Take Place »]. Studia Romanica Posnaniensia, Adam Mickiewicz University Press, Poznan, vol. XXXIII : 2006, pp. 316. ISBN 83-232-1643-6, ISSN 0137-2475.

The "Speech to the Dead" delivered by Hector in Jean Giraudoux's play The Trojan War Will Not Take Place (1935) exemplifies a cultural adaptation achieved by moving the relevance of the interwar period into the world of antiquity and the world of Homer. In 1935 Hector's speech expresses the protest of the whole generation against the First World War. But a closer look at this famous scene will demonstrate that Giraudoux actually defines the nature of the theatrical word which enables what is absent from the stage to appear on the stage, and which gets addressed to the unknown, mysterious interlocutors. Therefore the explication of these two tirades unveils a peculiar theory of the theatrical language.

Le discours aux morts que prononce, durant la scène 5 de l'acte II, le héros troyen Hector dans La guerre de Troie n'aura pas lieu, constitue l'une des scènes qui se rapprochent le plus de la récente actualité des spectateurs en 1935, soit l'après-guerre de $1918^{\circ}$. Car les discours de commémoration devant les monuments aux morts se sont multipliés, à l'issue de ce conflit que l'on appelle pour l'instant la Grande Guerre, et auquel Giraudoux a participé en première ligne. Pour comprendre le sens et la portée de ce fragment de scène, l'un des plus célèbres de la pièce, plusieurs points de vue sont à prendre en compte successivement.

Comment évoquer « la guerre de 1914 » au sein de La guerre de Troie n'aura pas lieu? Les deux événements se situent à une grande distance l'un de l'autre. La

" J. Giraudoux, Théâtre complet, édition établie, présentée et annotée par G. Teissier, préface de J.-P. Giraudoux, Le Livre de Poche, « La Pochothèque », 1991, pp. 514-515. 
tradition nous enseigne que la guerre de Troie, opposant une cité d'Asie mineure aux Achéens, a dû se situer au XII ${ }^{\mathrm{e}}$ siècle avant Jésus-Christ. Si le souvenir nous en est parvenu par les épopées d'Homère, que l'on situe quant à elles au VIII siècle avant notre ère, les fabuleuses découvertes de Schliemann ont procuré un fond historique inattendu à la légende homérique. Giraudoux joue sur ce mélange d'histoire et de légende (l'épopée, c'est l'histoire écoutée aux portes de la légende, avait dit Victor Hugo), pour retravailler les données du mythe et de la littérature grecque : la guerre de Troie pourrait avoir eu bien des causes, plus historiques que légendaires, puisque notamment dans la pièce, on a appris dès la première scène que Pâris et Hélène ne s'aiment peut-être pas.

Mais la plus grande liberté que prend le dramaturge avec la tradition culturelle occidentale, c'est de situer sa pièce, non du côté des vainqueurs venus de Grèce, mais des vaincus enfermés dans leur cité troyenne; et surtout, d'entamer l'action, non pas au cœur de la guerre et de la prise de Troie, qui constituent pour nous le tout premier événement raconté par une œuvre littéraire, mais juste avant cette origine même, puisque le titre de la pièce renvoie ce passé le plus lointain dans un proche avenir. Juste avant la guerre de Troie, a eu lieu en Asie mineure une guerre interne, dont Hector, vaillant fils du roi Priam, est revenu victorieux dans les premières scènes de l'acte I. Avant la première guerre de la littérature occidentale, il y avait déjà une autre guerre, qui dans la scène à commenter est en train de se clore. Il faut qu'Hector prononce un discours aux morts pour que l'on puisse fermer les portes de la guerre et que s'ouvre ainsi officiellement une ère de paix. Notre scène réveillera donc, chez le spectateur de 1935, une rumeur confuse de souvenirs scolaires, Homère, les tragiques et la civilisation grecs, mais décalés dans le temps et dans l'action.

Pour ce même spectateur cependant, la Grande Guerre n'est pas loin, et les anciens combattants restent nombreux. Giraudoux a pris une part directe au premier conflit mondial. Il a été blessé à la bataille de la Marne, puis sur le front des Dardanelles, dans ce détroit reliant la mer Égée et la mer de Marmara le long de la Troade. Là, il a pensé à ses souvenirs classiques et à Hélène, comme il en a témoigné : " Chacun de nous était caressé par un rayon, qui avait la longueur exacte de celui qui caressait Hélène. Nous étions au degré le plus doux du plus divin des thermomètres. Nous avions l'impression de nous servir du fard, de la poudre de riz d'Hélène ». Aussi ne doit-on pas être perplexe, au moment d'apercevoir le récent conflit mondial à travers la mise en scène d'une guerre antique : notre auteur a publié, durant les vingt dernières années, au moins trois livres sur la guerre : Lectures pour une ombre en 1917, Amica America en 1919 et Adorable Clio en 1920. Dès lors, La guerre de Troie n'aura pas lieu anime l'univers des Croix de bois de Dorgelès. Le titre lui-même de la pièce transpose le "plus jamais ça " lancé à l'issue du conflit ; Andromaque affirmant d'Hector (acte I, scène 1) : «il m'a juré que cette guerre était la dernière » traduit « la der des der » que devait être la guerre de 1914 pour les contemporains; quand Andromaque se montre prête à couper 
l'index de la main droite à Astyanax encore à naître (acte I, scène 3), pour préserver l'enfant d'aller plus tard à la guerre, elle évoque les mutilations des réformés qui se coupaient une phalange pour ne pas être envoyés sur le front; quand Demokos affirme (acte I, scène 6) : «Les guerriers qui n'ont pas un portrait de femme dans leur sac ne valent rien », le spectateur revoit l'image des soldats dans les tranchées ; ces soldats, on les saoulait avant l'assaut, comme ici le même Demokos prône pour les prochains combats «l'ivresse physique, que leurs chefs obtiendront à l'instant de l'assaut par un vin à la résine vigoureusement placé » (acte II, scène 4); on courait enfin le risque d'une fraternisation entre soldats ennemis dans les tranchées, que suggère dès le début (acte 1 , scène 3 ) Hector de retour du combat. Tous ces détails et débats sur la guerre éveillent chez les spectateurs du temps des images précises et un écho à l'état d'esprit immédiatement contemporain.

Hector, qui va prononcer ce discours aux morts, incarne dès lors l'ancien combattant désabusé. Dans Bella (1926), Giraudoux avait mis en scène Rebendart (comprenons, Poincaré) prononçant un discours pour inaugurer un monument aux morts. On peut considérer que le héros troyen apporte ici la réponse du dramaturge au chef d'État contemporain. Fermera-t-on les portes? se demande-t-on dans la scène précédente. Demokos tient conseil : sous les quolibets de la reine Hécube, il explique le rôle du poète au service de la guerre. Il prépare un chant de guerre et un concours d'épithètes. On va cependant fermer les portes de la guerre. Priam et Demokos manifestent leur hostilité. Busiris est consulté : son retournement donne lieu à un jeu de scène grotesque. Hector prononce alors le discours aux morts; au même instant, les Grecs arrivent en musique.

Cette scène se situe, structurellement dans la pièce, à mi-chemin entre la désillusion d'Hector exprimée au début à Andromaque dans la scène 3 de l'acte I et l'entrevue de négociation entre Hector et Ulysse dans la scène 13 de l'acte II ; avec cette dernière scène, $c^{\prime}$ est donc aussi celle qui donne le plus littéralement raison au titre de la pièce : le discours pour la paix, qui s'oppose au poème guerrier que rêve d'écrire Demokos, est prononcé au moment où Troie referme les portes de la guerre. Son contenu est marqué cependant par un anticonformisme fortement iconoclaste, dans lequel se joue une double parodie, celle de la civilisation antique et celle des discours officiels. Deux sentiments contraires s'y manifestent par là, chez Giraudoux écrivain : la tendresse pour le monde homérique, et en même temps la colère contre les gouvernements contemporains. Les deux tirades d'Hector, entrecoupées d'épisodes de stichomythie (on s'autorisera ce mot, même si le texte n'est pas écrit ici en vers, en tant qu'il rend compte des intermèdes par répliques rapides s'opposant aux masses des discours, tels que les a consacrés la tragédie classique en alexandrins), mettent en jeu les ressorts de l'humour et de la fatalité. Notons que le discours fait intervenir la rhétorique au théâtre, ce qui est rare chez Giraudoux, sinon à des fins de mise en scène parodique (le discours diplomatique de Busiris, patriotique de Demokos ou du Géomètre). Mais le discours place aussi au premier plan la parole, ce qui est conforme aux orientations de cette génération 
de dramaturges de l'entre-deux-guerres, qui conçoivent leurs pièces, en réaction au théâtre de boulevard du début du siècle, comme un théâtre d'idées dans un décor relativement nu, où le conflit des valeurs redonne de la profondeur au phénomène théâtral. C'est Jouvet qui a assuré la mise en scène sobre de la pièce, et qui tient ici le rôle d'Hector: il servira le texte de sa voix chaude, retenue et envoûtante, que Giraudoux a déjà dans l'oreille quand il écrit ces deux tirades.

HECTOR : Õ vous qui ne nous entendez pas, qui ne nous voyez pas, écoutez ces paroles, voyez ce cortège. Nous sommes les vainqueurs. Cela vous est bien égal, n'est-ce pas? Vous aussi vous l'êtes. Mais, nous, nous sommes les vainqueurs vivants. C'est ici que commence la différence. C'est ici que j'ai honte. Je ne sais si dans la foule des morts on distingue les morts vainqueurs par une cocarde. Les vivants, vainqueurs ou non, ont la vraie cocarde, la double cocarde. Ce sont leurs yeux. Nous, nous avons deux yeux, mes pauvres amis. Nous voyons le soleil. Nous faisons tout ce qui se fait dans le soleil. Nous mangeons. Nous buvons... Et dans le clair de lune! Nous couchons avec nos femmes... Avec les vôtres aussi...

DEMOKOS : Tu insultes les morts, maintenant?

HECTOR : Vraiment, tu crois?

DEMOKOS : Ou les morts, ou les vivants.

HECTOR : Il y a une distinction...

PRIAM : Achève, Hector... Les Grecs débarquent...

HECTOR : J'achève... Ô vous qui ne sentez pas, qui ne touchez pas, respirez cet encens, touchez ces offrandes. Puisque enfin c'est un général sincère qui vous parle, apprenez que je n'ai pas une tendresse égale, un respect égal pour vous tous. Tout morts que vous êtes, il y a chez vous la même proportion de braves et de peureux que chez nous qui avons survécu et vous ne me ferez pas confondre, à la faveur d'une cérémonie, les morts que j'admire avec les morts que je n'admire pas. Mais ce que j'ai à vous dire aujourd'hui, c'est que la guerre me semble la recette la plus sordide et la plus hypocrite pour égaliser les humains et que je n'admets pas plus la mort comme châtiment ou comme expiation au lâche que comme récompense aux vivants. Aussi, qui que vous soyez, vous absents, vous inexistants, vous oubliés, vous sans occupation, sans repos, sans être, je comprends en effet qu'il faille en fermant ces portes excuser près de vous ces déserteurs que sont les survivants, et ressentir comme un privilège et un vol ces deux biens qui s'appellent, de deux noms dont j'espère que la résonance ne vous atteint jamais, la chaleur et le ciel.

LA PETITE POLYXĖNE : Les portes se ferment, maman! HÉCUBE : Oui, chérie.

LA PETITE POLYXĖNE : Ce sont les morts qui les poussent?

HÉCUBE : Ils aident, un petit peu.

LA PETITE POLYXĖNE : lls aident bien, surtout à droite.

HECTOR : C'est fait? Elles sont fermées?

LE GARDE : Un coffre-fort...

HECTOR : Nous sommes en paix, père, nous sommes en paix.

HÉCUBE : Nous sommes en paix ! 
S'adressant aux morts, cet ancien combattant de la Grande Guerre déguisé en héros troyen n'en renoue pas moins avec les origines du théâtre grec. Celui-ci, on le sait, s'est constitué au bord des tombeaux, par dérivation de rites funéraires, des rites qui se déroulent sur de la terre pour communiquer avec le royaume des morts, et à ciel ouvert pour se tourner vers le monde des dieux. Des rites religieux qui préfiguraient définitivement la parole théâtre, qui prononcée en un moment précis n'en est pas moins valable pour tous les temps, et mise en scène en un lieu précis n'en incarne pas moins les lois du monde même : un maintenant mais pour toujours, et un ici valable pour tous les ailleurs.

Le ô lyrique qui ouvre la tirade détache ce moment de parole de toutes les paroles, cruelles ou simplement grotesques, qui ont pu précéder; il pose la solennité du discours, sa nécessité officielle, mais aussi la valeur poétique que peut prendre par moments la parole théâtrale. Ce discours sera donc une invocation, construite autour du pronom vous incessamment prononcé jusqu'à donner à toute la tirade sa coloration sonore. Mais une fois posés tous ces éléments de convention, toutes ces lois du genre, le discours aux morts butte d'emblée sur le paradoxe, refermé tout entier sur les négations (depuis le début de la pièce, en raison même de son titre, les personnages ont à discuter les valeurs des tournures négatives) : " vous qui ne nous entendez pas, qui ne nous voyez pas ». Giraudoux, animé d'un athéisme voire d'un anticléricalisme discret mais insistant dans toute son œuvre, met en scène la communication du monde terrestre avec un autre monde pour souligner essentiellement que cette communication n'existe pas. C'est une contestation à la base du discours aux morts comme pratique absurde qu'il met en avant, sans doute à la surprise du spectateur. Existe-t-il un monde purement intelligible hors de ce monde sensible, telle est la question métaphysique sous-jacente à la conjugaison tour à tour négative puis positive (écoutez, voyez) des verbes de sensation. Toute prière, dans cet univers cosmique mais matérialiste de Giraudoux, s'adresse à des dieux indifférents (1'indifférence des dieux sera un thème insistant dans Électre, dcux ans plus tard). À quoi Vigny avait répondu que «seul le silence est grand, tout le reste est faiblesse », quand l'Hector de Giraudoux souligne que l'homme qui parle aux morts, parle pour lui-même, c'est-à-dire à soi-même. À ce compte, tous les verbes changent de sens. Deux mondes se trouvent ainsi confrontés, celui absent du " vous » et celui présent du " nous », deux mondes dont les rapports ne vont cesser d'être définis, au cours de ce discours en deux tirades.

Ce «nous » de la terre, d'ici-bas, est remplacé par son action : «ces paroles », " ce cortège ». La parole théâtrale est nécessairement déictique, puisqu'elle ne peut faire exister le monde extérieur que par les mots. Ainsi le second substantif est-il inclus dans le premier : le cortège des Troyens rentrant victorieux dans leur cité ne peut être sur scène qu'un cortège de paroles; le cortège, action extérieure que le spectateur ne peut voir, s'absorbe dans les paroles qui seules peuvent se dérouler 
sur scène. Le «nous » assoit cependant sa position, face au «vous» des absents : "Nous sommes les vainqueurs ». Un souvenir de la civilisation antique passe ici devant les yeux du spectateur, celui du défilé dans la cité du général victorieux après les guerres (on parlera à Rome d'imperator et de triomphe). Les vainqueurs le sont ici de cette guerre interne en Asie, qui a précédé immédiatement celle qui sera dite de Troie par les générations futures. C'est dire que la roche tarpéienne est près du Capitole, puisque les vainqueurs d'aujourd'hui vont, aujourd'hui et demain, soit périr soit être emmenés en captivité par les nouveaux « vainqueurs ». C'est dire que l'article défini revêt, pour les désigner, une valeur contradictoire, à la fois universelle et dérisoire : ce sont les vainqueurs, comprenons de tous les temps, mais aussi bien d'aujourd'hui seulement.

Jouant constamment à faire alterner conventions et anticonformisme, à peine Giraudoux a-t-il mis en place la situation attendue d'un discours officiel qu'il réintroduit la dissonance par une question inattendue : "Cela vous est bien égal, n'est-ce pas ? \Cette indifférence invalide de fait la solennité des vainqueurs. Face au pathétique de la situation, l'indifférence construit cet univers binaire propre à toute l'œuvre de Giraudoux, depuis le roman autobiographique Simon le pathétique (paru en 1918). De même dans la pièce, Andromaque est pathétique, comme elle l'était dans la pièce de Racine, mais Hélène est indifférente. C'est bien cet antagonisme entre le pathétique et l'indifférence qui accentue le clivage entre le "vous » et le «nous»: «Vous aussi, vous l'êtes. Mais, nous, nous sommes les vainqueurs vivants ». Cette petite guerre verbale à coups de pronoms toniques et de $v$, joue sur la mention "Morts pour la patrie»; pensant avec Gide et contre Demokos que ce n'est pas avec de bons sentiments qu'on fait de bonne littérature, Giraudoux introduit la lame de son couteáu dans l'esprit bien-pensant et respectueux des survivants, pour les mettre en face d'un sentiment inavoué : une joie revancharde, qui éclate dans la surprise de l'épithète finale vivants. La parole des écrivains se pose comme complémentaire de la parole officielle, comme propre à écrire, dirait Balzac, l'envers de l'histoire contemporaine. Le dramaturge moderne utilise le matériau des discours nationaux comme les auteurs classiques recouraient aux épisodes de l'histoire antique : pour écrire, dans les interstices de ces textes généraux et collectifs, l'histoire des enjeux et sentiments personnels.

$\mathrm{La}$ rhétorique réaffirme cependant ses droits : «C'est ici que commence la différence ». La notion de différence va servir à distinguer ce que l'on confond, et donc nourrir l'anticonformisme de la tirade. L'anaphore rhétorique «c'est ici» transforme le discours officiel en acte d'accusation. L'adverbe ici montre bien que, dans une pièce de théâtre, le lieu scénique et le moment de l'action ne sont qu'un point virtuel dans la marche d'une démonstration, qui sera l'enjeu profond de la pièce. Comme l'adjectif «vivants » il y a un instant, le mot «honte » maintenant accentue l'anticonformisme à l'aide d'un mot en coup de poing en fin de phrase. Voilà cependant que l'orateur s'est distingué de la masse du «nous » par un «je » qui va de plus en plus s'entendre comme énonçant la profession de foi de Girau- 
doux lui-même : «C'est ici que j'ai honte. Je ne sais si dans la foule des morts on distingue les morts vainqueurs par une cocarde ». La foule des morts incarne un instant l'Hadès de l'Antiquité mis en scène successivement par Homère puis Virgile, ce qui accentue la dissonance de la mention toute contemporaine et française de la cocarde. On le voit, dans cette tirade, les propos malsonnants sont secondés par l'esthétique de la dissonance. L'anachronisme, qui se glisse dans bien des répliques de la pièce (on a pris une photographie dans la scène 3 de l'acte II), constitue le biais par lequel Giraudoux se rend maitre du mythe ; il rappelle au spectateur qu'un auteur se cache derrière le personnage; il brise enfin l'illusion scénique, comme on le reverra plus loin.

La rhétorique d'Hector procède par divisions binaires : s'adresser aux morts, c'est donner à voir et à entendre. Être vainqueur, on peut l'être mort ou vivant. Et chez les morts, distingue-t-on les vainqueurs à la guerre de tous les autres? Question non uniquement rhétorique d'ailleurs, qui met l'accent sur l'absurdité éventuelle des idées reçues : les morts au combat ont-ils un monument dans l'audelà ? La dichotomie travaille dans le détail toute la pièce, laquelle a précisément pour objet de déterminer si la guerre de Troie aura lieu - ou pas. Une nouvelle alternative s'articule cependant aux précédentes : «Les vivants, vainqueurs ou non, ont la vraie cocarde, la double cocarde. Ce sont leurs yeux ». $\grave{A}$ ces survivants pourvus d'yeux encombrants s'opposent les armées aveugles qu'évoquait Hector à Andromaque, dans la troisième scène de la pièce : même si les mères, pour préserver leur fils du combat, « lui crèvent les yeux, les armées seront aveugles, mais il y aura des armées ». Une idée de culpabilité s'attache bien sûr à ces yeux : songeons à la légende d'CEdipe. «Nous, nous avons des yeux, mes pauvres amis ». Le sentiment humain rapproche le monde d'ici-bas et l'hypothétique au-delà.

Hector en tire les conséquences : «Nous voyons le soleil ». L'expression est communément admise au sens simple de vivre. Dans le vocabulaire d'Hector, elle désigne la manière troyenne de vivre, puisque face à Ulysse, dans la scène des pesées, il déclarera : " Je pèse le faucon, je regarde le soleil en face 》 (acte II, scène 13), ce qui n'est pas sans rappeler le mythe d'Icare, et ce à quoi Ulysse répondra : "Je pèse la chouette ", celle d'Athéna. De même ici la mention du soleil amène-t-elle celle du "clair de lune ". Par là, le destin de l'homme reste toujours relié à l'univers chez Giraudoux : c'est même à ce signe que l'on reconnaît que le destin est en jeu. Le discours aux morts devient logiquement, puisqu'on ne sait rien des morts, un discours sur les vivants : «Nous mangeons, nous buvons ». La mort est une mise en perspective, par rapport à laquelle même les détails les plus quotidiens se trouvent transfigurés, notamment après un désastre. Le quotidien, c'est aussi ce par quoi les dramaturges comme Giraudoux puis Anouilh rénovent la mise en scène des mythes antiques. Giraudoux notamment en a témoigné dans Simon le pathéti$q u e$ : autrefois, « les fils et les filles doués étaient évidemment envoyés à la préfecture, mais dans un lycée fermé de grilles où ils étaient mis aussitôt en rapport direct 
et exclusif avec l'antiquité, et coupés plus encore du monde ». Le quotidien, dans ses pièces, rétablit au contraire le lien entre l'Antiquité et le monde.

En fait, les alternatives, selon le rythme naturel de la tirade, se chevauchent : le soleil et la lune, manger et boire. La seconde est englobée par la première, qui reprend comme après un arrêt : «... Et dans le clair de lune !...», ce qui donne à la tirade l'apparence d'une improvisation structurée. Sur la scène de Giraudoux où tout passe sans qu'on s'y attarde, l'érotisme est toujours rapide et allusif: «Nous couchons avec nos femmes... ", ce qui évoque l'atmosphère d'intimité conjugale de la troisième scène de la pièce où l'on voyait Hector rendu à sa femme après trois mois de guerre, mais qui introduit aussi la provocation finale : «Avec les vôtres aussi », qui remet en question la moralité des après-guerre et suggère l'idée subversive selon laquelle les morts au chant d'honneur ont été, d'abord au sens figuré, des « cocus ». C'était déjà le sujet d'Amphitryon 38, et la prochaine guerre de Troie a bien pour origine une affaire de cocuage.

Alors commence une rapide séquence de stichomythie, comme un intermède, un entracte, entre les deux parties du discours d'Hector. À la représentation, le poids respectif de ces interventions est souligné par l'organisation spatiale sur scène: Hector parle face aux portes ; la voix de Demokos vient d'un côté, celle de Priam de l'autre, symbolisant comment ce face-à-face métaphysique interfère avec le débat interne dans la cité (premier intervenant) et l'urgence extérieure (second intervenant).

Demokos, représentant du parti des bellicistes, personnage créé par Giraudoux, a reçu un nom formé sur dèmos, le peuple, d'où l'on tire démagogie, et kakos, mauvais. Dans son indignation : «Tu insultes les morts, maintenant? », il représente certainement une partie du public, et Hector une autre partie. Conventionnel, le poète ne supporte pas l'inversion des valeurs qui est dénoncée, et que suggère à nouveau avec ironie son interlocuteur: «Vraiment, tu crois? » D'où résulte un quiproquo: la question d'Hector portait sur le fait d'insulter. Mais la remise en question des valeurs reçues serait trop profonde; Demokos opte pour une interrogation portant sur les morts : «Ou les morts, ou les vivants », ce qui souligne, aux yeux d'un spectateur plus réfléchi, la complexité des après-guerre, trop pressées de trancher entre héros et traîtres, et permet à Hector de reprendre le mécanisme de ses dichotomies dialectiques : "Il y a une distinction... ». Priam, le roi âgé de Troie, dont le rôle reste effacé en regard du patriotisme national de son fils, demeure audessus des partis : "Achève, Hector... Les Grecs débarquent... » Garant de l'État, le roi est celui qui veille au plein accomplissement des rites, y compris religieux (Georges Dumézil a montré comment une société indo-européenne réunit dans une "première fonction " le pouvoir politique et religieux); Priam l'a précisé plus haut dans la scène, réclamant à Hector le discours aux morts : "La cérémonie le comporte. Le général victorieux doit rendre hommage aux morts quand les portes se ferment ". Dans Littérature, Giraudoux réfléchit au rôle du roi dans le théâtre classique : c'est pour le définir comme le personnage moyen et universel. Le roi ici 
en revanche ne sait pas que le destin a choisi ce même Hector pour achever en effet l'action en déclenchant en dernier lieu la guerre. La mention des Grecs provoque une dramatisation de la scène, comme une menace qui sera très bientôt renforcée par une indication scénique : «La musique des Grecs éclate », au moment où les portes de la guerre se seront refermées. Le spectateur de 1935, mieux que tout autre, sait lire dans cette situation pressante le tragique et la montée des périls d'un entredeux-guerres. L'espace extérieur à la scène, présent ici dans la réplique, donne à voir un rivage, et crée un lointain poétique que Giraudoux hérite de l'univers racinien.

La seconde tirade d'Hector adopte, telle une strophe, le même rythme que la première, pour développer le même paradoxe. Seul le début a changé : " J'achève... », qui doit retenir l'attention, car il signifie que dans cet univers, les paroles sont comptées aux hommes, à la façon d'un compte à rebours. Les mots s'écoulent comme dans un sablier vers un terme marqué d'avance : c'est ce qui donne à leur substance une intensité tragique sans gratuité ni innocence possibles. L'univers intelligible est renvoyé à son absence de sensations : «Ô vous qui ne sentez pas, qui ne touchez pas "; deux sens nouveaux, comme si la confrontation des vivants et des morts se faisait par leur recensement méthodique. Sentir pourrait être synonyme de toucher, mais le verbe respirer confirme qu'il s'agit bien de recenser aussi l'odorat. Le rappel de la religion antique se fait plus précis : "respirez cet encens, touchez ces offrandes », univers de la religion que développera bientôt Électre, univers des offrandes funéraires qui sera au cœur de l'Antigone d'Anouilh (1944). Ici comme tout à l'heure, les impératifs ont été par avance frappés de vanité par les relatives. Le texte prononcé suppose la présence correspondante des objets sur la scène, à moins que la recherche d'un spectacle épuré ne réduise ce rite cultuel à des gestes symboliques. Après les répliques brèves qui s'interrompaient les unes les autres, la rhétorique à long terme reprend ses droits, «puisque enfin c'est un général sincère qui vous parle ». Le général victorieux, c'est bien l'imperator romain, intercalé dans cette scène elle-même au voisinage de la civilisation grecque. Quant à la sincérité d'Hector, elle s'oppose aux paroles vides ou trompeuses des bellicistes : les deux camps sont bien marqués.

À nouveau, la dissonance s'introduit par surprise dans le panégyrique : «apprenez que je n'ai pas une tendresse égale, un respect égal pour vous tous ». Tout à l'heure, la fausse note consistait à demander : «Cela vous est bien égal, n'est-ce pas ? ». Le mot a changé de sens, mais d'un contexte à l'autre, la question centrale du discours est bien de savoir entre quoi et quoi on peut ou on ne peut pas proposer le signe mathématique égale. Les notions confrontées sont ici le courage et la mort, la vie et l'héroïsme, au moyen d'une vérité grinçante : "Tout morts que vous êtes, il y a chez vous la même proportion de braves et de peureux que chez nous qui avons survécu ». Ainsi s'instaure un mouvement de déculpabilisation qui vient compenser l'image des yeux cocardes. Car si les morts ne sont pas tous des héros, les survivants ne sont pas tous des traitres. Le bipolarisme du courage et de la 
lâcheté constitue un enjeu majeur dans la pièce : devant Oïax, le courage par exemple peut être de se laisser gifler (acte II, scène 9); et devant l'ennemi, la vaillance peut coïncider avec un mouvement de tendresse humaine (acte $I$, scène 3); enfin le fuyard n'est qu' ' un héros qui détale» (acte I, scène 6) : dans cette dernière scène, toute une discussion entre Andromaque et Priam a porté sur le faux clivage entre courage et lâcheté, ou entre héroïsme et traîtrise ; enfin l'ancien combattant est tour à tour défini, au cours de la pièce, comme peut-être un lâche (acte I, scène 6) puis comme un héros qui a tous les droits (acte II, scène 5) : «Cela devient impossible de discuter l'honneur avec ses anciens combattants. Ils abusent vraiment du fait qu'on ne peut les traiter de lâches », ainsi que le regrette Demokos. Dans cette situation complexe, Hector et ses dichotomies introduisent le facteur de nuance ( vous ne me ferez pas confondre »). La cérémonie ici en jeu justifie après coup la forme rhétorique du discours; elle renvoie aussi bien au rituel antique qu'aux commémorations officielles modernes; elle revêt aussi bien une valeur de satire.

De fait, le spectateur à la fin de la pièce, tout comme Hector en cet instant et par principe, ne confondra pas les morts à admirer et les morts qu'il n'admirera pas. Ces derniers se trouveront dans les deux camps : Ö̈ax du côté grec, Demokos du côté troyen. Ces considérations complètent celles sur le faux clivage entre courage et lâcheté : la guerre peut aussi produire un héroïsme abject. Contrairement au Créon qu'animera neuf ans plus tard Anouilh, Hector refuse ainsi qu'une fonction officielle conduise nécessairement aux compromissions : "vous ne me ferez pas confondre », "ce que j'ai à vous dire aujourd'hui ». Aujourd'hui porte la tension tragique à son comble. Les morts, auxquels l'orateur n'accorde pas d'existence audelà du sensible, représentent néanmoins une force dans le monde sensible, presque une puissance trompeuse, aurait dit Pascal. Le héros dialogue cependant avec eux, comme avec des interlocuteurs plus privilégiés : ils sont médiateurs de vérités que les humains ne sauraient entendre directement. À la faveur de ce dialogue paradoxal, la scène se fait tribune, et la tirade pamphlet contre la guerre : « la guerre me semble la recette la plus sordide et la plus hypocrite pour égaliser les humains ». Ainsi se nuance un sens possible du titre de la pièce : la guerre de Troie n'aura pas lieu, au sens où elle ne doit pas avoir lieu. Chemin faisant, notons que cette condamnation fait retour une troisième fois sur la question du signe égale.

A l'approche de la péroraison du discours, la rhétorique se fait plus voyante, c'est-à-dire la syntaxe plus élaborée, par l'enchevêtrement des subordonnées : «j'ai à vous dire aujourd'hui (...) que je n'admets pas plus la mort comme châtiment ou comme expiation au lâche que comme récompense aux vivants ». Ce faisant, le sujet des deux tirades est repris, sous forme de synthèse, en chiasme. La guerre est essentiellement signe de contradiction. L'adverbe aussi, amorçant la conclusion au point de vue de la construction logique, répond indirectement à la demande de Priam d'achever le discours à l'arrivée des Grecs. Le paradoxe propre au discours destiné aux morts est renforcé par les structures concessives (tout morts que vous 
êtes, qui que vous soyez), qui permettent de cerner au plus près la nécessité de cette parole pourtant sans destinataires connus et peut-être possibles : il faut parfois parler, même si l'on ne sait pas à qui, et il faudra revenir en conclusion sur ce problème de destination autour duquel est construit tout le discours d'Hector.

Comment définir, donc comment se représenter l'au-delà ? C'est sur cette question que va se clore l'allocution aux morts. Elle cheminera à travers un rythme ternaire, alliant les mots vous et sans. Défini par rapport aux vivants, l'au-delà est placé sous le signe de la coupure; par rapport aux morts, il confine à l'absurde : « vous absents, vous inexistants, vous oubliés, vous sans occupation, sans repos, sans être ». Ainsi se déroule une sorte de litanie privée de son contenu religieux, puisque sans repos s'oppose à la formule consacrée le repos éternel. La contradiction travaille même le statut des morts, puisque les uns sont ressentis comme absents, les autres au contraire oubliés, ce qui nous ramène aux deux versants de l'univers giralducien, l'indifférence et le pathétique. L'au-delà est ici cerné comme ce qui échappe à la pensée humaine, comme le domaine sur lequel la représentation n'exerce aucune prise. Mais pensé au plan humain, l'au-delà commue ses valeurs en inquiétudes discrètes : la sérénité (du Paradis ?) se fait absence d'occupation, c'est-à-dire peut-être ennui ; l'éternité (du purgatoire ?) se fait absence de repos, c'est-à-dire peut-être tourment.

C'est ici la parole qui commande l'action, puisque le discours se conclut « en fermant ces portes », ce qui était son objet et son but. La parole exerce aussi son action sur les valeurs reçues, qu'elle annule au fur et à mesure qu'elle avance : au moment d'«excuser près de vous [les morts] ces déserteurs que sont les survivants », excuser contredit toute glorification a priori contenue dans une cérémonie commémorative, et survivants est annulé par déserteurs, dans une définition que rend originale le point de vue adopté, comme au moment, on l'a vu, de définir le lâche " un héros qui détale ». De même, pour ces survivants, « ressentir comme un privilège et un vol » la sensation et la perception de la chaleur et du ciel leur donne un statut ambigu, vol annulant privilège. La chaleur et le ciel, qui constituent d'abord « deux biens » réservés aux survivants, deviennent pour les morts « deux noms », dans cet univers théâtral où les mots priment les objets : c'est le mot cocu, appliqué à Ménélas, qui déciderait de la guerre (comme dans Électre, les mots adultère et parricide décident du meurtre), la guerre qui a engendré les mots de courage et de lâcheté ; et dans la présente situation, si « la guerre exige un chant de guerre » (acte II, scène 4), la paix exige un discours aux morts. Ainsi les sensations de la vie se réduisent-elles pour les morts à «deux noms dont $j$ 'espère que la résonance ne vous atteint jamais », ce qui rappelle le rôle du bruit et de la musique dans la pièce (dans un instant, nous apprendrons que «la musique des Grecs éclate »). La chaleur prolonge en sensation tactile l'image du soleil, et le ciel, sur lequel le discours semble ouvrir pour finir, a été nié en tant que réalité métaphysique, et ne demeure que comme l'azur poétique d'un paysage antique. 
Suit une nouvelle séquence de stichomythie, un dialogue entre plusieurs membres de la famille royale troyenne. La petite Polyxène, la plus jeune fille de Priam et d'Hécube, n'apparaît dans la littérature antique qu'après l'Iliade. Sacrifiée à l'instigation d'Ulysse pour favoriser le retour, elle offre un équivalent d'Iphigénie. Ici, il lui revient d'introduire sous forme de dialogue une indication scénique qui fait exister par la seule parole l'univers hors de la scène : «Les portes se ferment, maman! » Le langage enfantin («Oui, chérie », lui répond Hécube) introduit dans la scène un sourire après la gravité. Seconde épouse de Priam, Hécube se signale par sa fécondité, ayant donné le jour à dix-neuf enfants dont plusieurs sont les personnages de la pièce. Elle joue un rôle effacé dans l'Iliade, et symbolise la majesté et le malheur chez les tragiques, lorsqu'elle sera confiée à Ulysse pour sa captivité. Mais le court dialogue entre mère et fille (qui semblent ici grand-mère et petite-fille) complète la remise en question des représentations religieuses opérée par Hector : "-Ce sont les morts qui les poussent. - Ils aident, un petit peu. - Ils aident bien, surtout à droite "). L'idée suggérée est qu'une religion (le christianisme dans la civilisation occidentale) se réduit à un conte pour enfants, et notamment un moyen de rendre concrète et donc rassurante la perspective de la mort. Le « un petit peu » d'Hécube suggère une concession à cette représentation commode; et le «bien » de Polyxène l'efficacité de l'astuce. Le comique du dialogue («Ils aident bien, surtout à droite ») prend ainsi discrètement une valeur iconoclaste. Il ménage en outre dans la scène une variété de tons, après la gravité des enjeux précédents. Dans la pièce Siegfried (acte I, scène 8), le personnage demande : «C'est une comédie, une tragédie? ", à quoi Robineau répond: «Tous les genres se mêlent dans le théâtre moderne "; et dans Littérature, après avoir souligné les liens secrets reliant la tragédie à la farce (c'est bien le cas ici), Giraudoux définit « les comédies les plus modernes ou les plus frivoles » comme «un voile léger jeté sur des voix et des silhouettes tragiques ». Mais en outre, il faut que les portes se ferment, et le comique chez Giraudoux joue à ce titre assez souvent un rôle d'intermède. Le personnage d'Intermezzo précisément le dit : «Je n'ai absolument rien à faire. Je reste une minute, pour la transition 》.

C'est Hector qui la clôt, en ramenant la gravité : "C'est fait? Elles sont fermées? $»$ L'artisan de la paix donne au titre de la pièce un instant fort de consistance : la guerre n'aura pas lieu. Fermer les portes de la guerre constitue, sous la plume de Giraudoux, un anachronisme discret, puisqu'il s'agit d'un rite romain et non grec ni à plus forte raison troyen; le personnage du général victorieux avait préparé le spectateur à ce décalage de civilisation. Le garde qui prononce alors une courte réplique : «Un vrai coffre-fort » pose, par sa seule présence, Priam et Hector dans leur rôle de commandement. Comme la servante de la toute première scène, ou les gabiers qui ont accompagné Pâris lors de l'enlèvement d'Hélène, il représente sur scène le monde extérieur. La comparaison de la cité close avec un coffre-fort, elle aussi discrètement anachronique, suggère bien évidemment ici que la paix est un précieux trésor. Mais l'image revêtira un sens plus profond à long terme. Troie est un coffre-fort, parce qu'il renferme des richesses. Plus loin (scène 16), lors de la 
grande confrontation entre Hector et Ulysse, le premier lancera au second, venu en ambassade réclamer Hélène : «Avouez-le. Vous voulez nos richesses ! Vous avez fait enlever Hélène pour avoir à la guerre un prétexte honorable ! » Ce qui suggère, tout au long de la pièce, toute une réflexion anthropologique sur la constitution des mythes comme réinterprétation de l'histoire économique : pour l'historien, l'enlèvement d'Hélène aurait été inventé par les littérateurs travaillant sur le souvenir d'une cité détruite par la civilisation grecque en pleine expansion commerciale.

Un père, une mère, un fils aîné et une fille cadette assistent à cette scène officielle : Giraudoux voulait ajouter, à la règle des trois unités mise en œuvre dans la tragédie classique, une unité de famille qu'il trouve dans les pièces de Corneille. Ce sont bien ces rapports familiaux qui, au moment où les portes de la guerre se ferment, tissent la cohésion de l'univers tragique.

Mais pour l'instant, les portes de la guerre se sont refermées sur une paix qui trouve son prix dans la menace. Prononcée d'abord par Hector, l'exclamation: «Nous sommes en paix, père, nous sommes en paix » exprime le rôle du héros comme bras droit de l'autorité pour assurer la survie d'une cité : le fils guerrier s'en remet au monarque pour consacrer la paix. Le voisinage même des mots paix et père définit le rôle pacificateur des rois dans la constitution politique des tragédies. Reprise par Hécube, la formule : « Nous sommes en paix ! » dégage la signification humaine de la situation pour les femmes et pour les familles : non plus la sûreté de l'État, mais la douceur du foyer. Ces deux exclamations renferment certainement une intense émotion pour un spectateur de 1935 ; prononcée juste une seconde avant que «la musique des Grecs éclate », elle prend une valeur de fort avertissement, et sonne aussi comme un cri pathétique et vain lancé dans un entre-deux-guerres.

Dans Le petit journal du 22 novembre 1935, Pierre Veber écrivait : « La guerre de Troie n'aura pas lieu n'est pas à proprement parler une pièce de théâtre. C'est plutôt une 'moralité légendaire', une construction philosophique où l'on fait le procès de la guerre ».

Moralité légendaire à portée philosophique et procès de la guerre, cet extrait de scène l'est certainement. On voit maintenant avec quelle légèreté et quel respect en même temps, avec quelle gravité et quelle insouciance, Giraudoux use du fonds homérique pour passer la situation la plus contemporaine de lui au filtre du patrimoine le plus lointain. L'Antiquité lui permet ici de porter sur la scène un débat assurément brûlant de l'actualité, par une combinaison de deux héritages, l'un mythique et originel (la guerre de Troie), l'autre récent et à tout jamais marquant (la Grande Guerre). Le dramaturge moderne met en scène une histoire connue, mais en y introduisant des inventions imprévisibles, à la source de dissonances, de sourires culturels, d'anachronismes discrets et d'une profonde réflexion moderne.

La dénonciation domine assurément dans ce discours aux morts, à travers une rhétorique qui dénonce la rhétorique des discours officiels, et plus discrètement 
celle des croyances ancestrales. Le rôle dévolu aux écrivains semble, au point de vue de Giraudoux, de dire ce qui gêne, d'introduire dans la vie politique et les valeurs reconnues cet esprit de distinction qui fissure les faux semblants et les subterfuges trop commodes.

Mais ici, la scène ne se réduit pas à une tribune (ce qu'a constitué entre autres, depuis des siècles, le théâtre : un moyen de dire au public du temps ce que l'on pense). Le discours aux morts intervient bien dans une pièce de théâtre, il ressortit à l'écriture théâtrale. Le lieu scénique se construit fortement sur la tension : celle des conflits internes, celle aussi de la pression extérieure. La déclaration de paix s'accompagne d'une montée des périls. L'unité cornélienne de famille renforce le pathétique de la destinée tragique. Entreprise de mort et ferment de contradictions, la guerre elle-même sert l'univers tragique et n'est pas loin de proposer au spectateur une métaphore du théâtre. Grande figure du paradoxe, elle construit un affrontement de valeurs : entre la guerre et la paix dans l'optique de Tolstoï, mais aussi entre l'individu et la société, et même entre le Droit et la justice ; elle nourrit l'affrontement des groupes : Troyens et Grecs, mais ici bellicistes et pacifistes, et de façon générale ceux (comme Hector) qui s'inscrivent contre la marche du destin, et ceux qui aveuglément l'accomplissent.

Figure du paradoxe, la guerre doit donc se clore par un discours aux morts que prononce Hector, un discours qui pose la parole même au théâtre comme paradoxe. Rhétorique contre la rhétorique, le discours d'Hector (lui-même en guerre contre la guerre) est marqué par l'époque de ce théâtre de l'entre-deux-guerres où, contre l'avis d'Antonin Artaud, c'est le texte qui fait la substance d'une pièce, au service d'un théâtre littéraire (Sartre conservera encore cette conception, dans la génération suivante). Mais quelle ambiguité dans le statut de ce texte! Hector venait de l'annoncer : «Il n'y aura pas de discours aux morts », si bien que les négations qui se multiplient dans ses répliques posent son discours comme un non-discours, et sa parole comme une non-parole. Son discours, ajoutait le guerrier devant Priam et Demokos, il l'a déjà prononcé, par fragments, sur le champ de bataille, devant chaque mort un à un, si bien que ses tirades d'aujourd'hui offrent l'artifice d'un texte trop continu et lisse, en malheureuse harmonie avec les destinataires eux aussi anonymes et absents auxquels il s'adresse à présent. Et de fait chez Giraudoux, la courte réplique est beaucoup plus porteuse de vérité que les tirades, à valeur presque exclusivement satirique. Les morts n'entendent pas les discours qu'on leur adresse, comme les hommes d'État de 1935 n'entendront certainement pas les avertissements que leur prodigue Giraudoux par la voix d'Hector. Mais de cette inutilité dérisoire, la parole théâtrale sort elle-même grandie, car elle aussi, prononcée sur une scène, ne s'adresse pas essentiellement aux interlocuteurs qui la reçoivent dans le lieu scénique. Au théâtre, c'est pour d'autres que l'on parle, la salle et non les personnages sur scène, et au-delà de la salle tout l'avenir des lecteurs : et ce public, en effet composé d'absents devant la scène, assurera seul la survie de l'univers ainsi créé. 